\title{
Correlation of Alzheimer's Disease with Wnt Signaling Pathway and Neural Stem Cells
}

\author{
Hongbo Yao, Lihui Sun, Jie Lian, Meng Zhang, Danyang Liu * \\ Qiqihaer Medical University, Qiqihaer City Jianhua District, 161006 \\ hunter2011@foxmail.com
}

Keywords: Alzheimer's Disease, Wnt Signaling Pathway, Neural Stem Cells.

\begin{abstract}
Recent studies have shown that Wnt signaling molecules and the occurrence and development of Alzheimer's disease are closely related. Wnt protein secretion by autocrine or located next to the cell membrane receptor binding, activation of intracellular signaling pathways to regulate expression of target genes of cell proliferation during embryonic development, differentiation, migration, polarity and apoptosis has played an important role. The pathological mechanism of Alzheimer's disease involves inherited, environmental and immunological factors. Strong evidence exists that Wnt signaling pathway is closely related to the pathological process of Alzheimer's disease. There is also evidence that Wnt signaling pathway plays a crucial effect on the proliferation of neural stem cells. To review the relationship between pathological process of Alzheimer's disease and Wnt signaling pathway and effects of Wnt signaling on proliferation and differentiation of neural stem cells, providing theoretical evidence for treatment of Alzheimer's disease.
\end{abstract}

\section{Theoretical Introduction of Alzheimer's Disease}

Alzheimer's disease (Alzheimer's disease AD) is more common in people over the age 60, Alzheimer's disease is the onset before the age of 65, and senile dementia refers to over 65 years of onset. AD etiology is not clear, family history is a risk factor for $\mathrm{AD}$, but also may be related to a number of diseases related to the immune system. Ap accumulate a large number of nerve cells and Wnt ligands will Ap direct binding, may also be combined with the cell membrane Fz receptors results in inhibition of Wnt signaling pathway, sometimes they exhibit relatively activation, inhibition of abnormal activation and signal causes cellular signal transduction direction changes, is blocked to intracellular signals transduced cells.

Its pathogenesis is currently elusive, the following theory as genetic mutation theory, theory of cholinergic nerve abnormalities, prisoners ingot amyloid, genetics, said the doctrine of central neurotransmitter metabolism, cytoskeletal change doctrine, the doctrine of immune abnormalities, free radicals and apoptosis in doctrine and excitatory amino acid toxicity theory from different angles, etc. are proved with Alzheimer's disease have a certain relevance. AR doctrine which dominates the hypothesis that AR prisoners ingot amyloid protein precursor (APP) cleavage is the main component of the brain cortex and hippocampus A Ll region form a large number of patients with senile plaques (senile plaque, SP) of It caused pathological changes of AD, leading to neurofibrillary tangles (neurofibrillary tangles, NFT) formation, as well as diffuse intracellular protein Tau River, vascular injury and dementia form ${ }^{[1]}$.

The key molecule Wnt signaling pathway is Ap, if activated, could play a role in prevention and treatment of $\mathrm{AD}$, this method is the use of signal molecules in specific pathways, activation of downstream effector to play. Current AD treatment methods for drug treatment: (1) for cholinergic treatments, including choline cool inhibitors and cholinergic agonists; (2) anti-prisoners ingot amyloid peptide drugs, including drugs to inhibit the formation of AR scattered, AR inhibition San neurotoxic drug, pro-AR bulk drug degradation; (3) radical scavengers; (4) estrogen therapy River; (5) herbal treatment called; (6) cell transplantation. It is currently in the focus of research of neural stem cells (Neural stem cells, NSCs) transplantation therapy AD. 


\section{Wnt Pathway Treatment Principle}

The composition of the Wnt signaling pathway include: extracellular factor W nt, transmembrane receptor frizzled (Frizzled), scattered protein (Dsh), adenomatous polyposis coli gene product (adenomatous polyposis coli, APC), glycogen synthase kinase -, Tam catenin (Khan catenin), axis protein (Axin) and $\mathrm{T}$ cell factor / lymphoid enhancer factor ( $\mathrm{T}$ cell fatorlymphpid enhancing factor, TCF / L EF) and so on. Wnt signaling pathway in regulating NSC, one of the important ways to the growth and proliferation, it is also involved in the control NSC, amplification and differentiation determine their final fate. cate-nin accumulate in the cytoplasm in normal cells may be blocked by the gene product. Serine / threonine kinases, USK-3 capable of APC gene and protein scaffold shaft was taken to a nearby target Khan catenin. N-terminal Khan catenin is USK-3 phosphorylation, thereby identifying the ubiquitin-proteasome-mediated degradation and promote their destruction ${ }^{[2]}$. Fig.1 shows the Wnt pathway treatment principle.

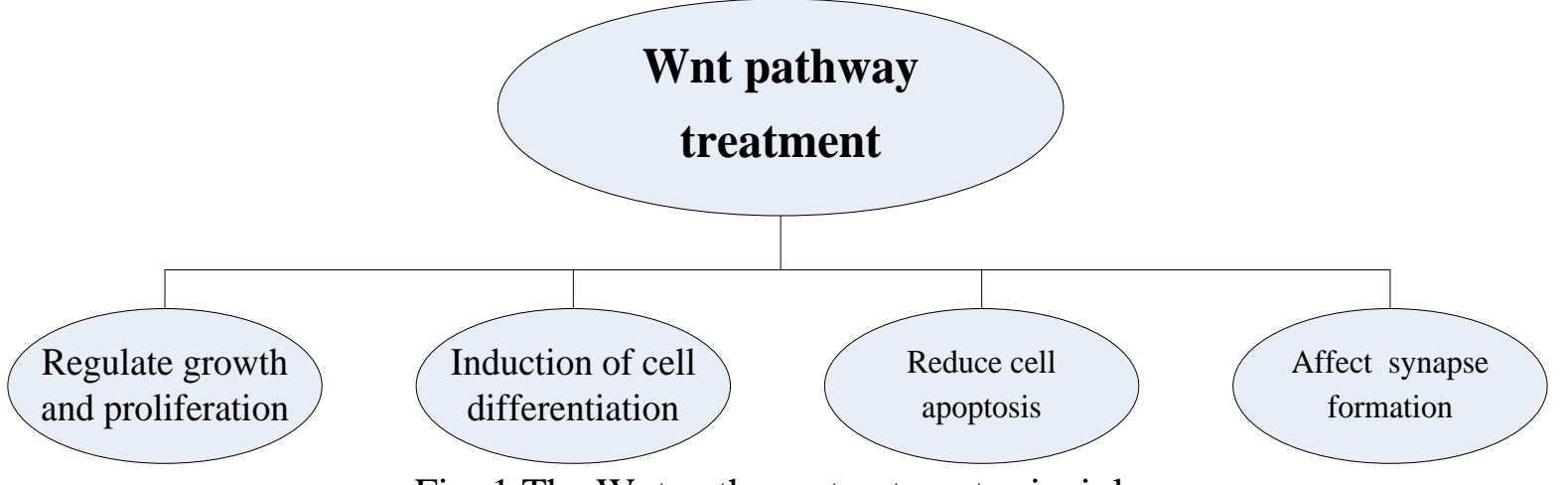

Fig. 1.The Wnt pathway treatment principle

Effect of Wnt signaling pathway by neural precursor cells in the hippocampus of adult stem cell research, using in situ hybridization found that Wnt close proximity SUZ, by the adult hippocampal astrocytes express, from hippocampal stem cells capable of expressing the receptor $\mathrm{W} n \mathrm{nt}$ and $\mathrm{W} \mathrm{nt}$ pathway signaling components mo. Cytokines hippocampal astrocytes produce induced pluripotent precursor cells (AHPs) to neuronal differentiation. W nt signal can be seen by a factor of astrocytes participation to hippocampal neurogenesis the proliferation and differentiation of neurons. Wnt signaling in the nervous system, blood vessel formation by analyzing TOP-UAL / Wnt mouse pointer, discovery and development, the classic W nt signal C'NS is specifically activated. However, early tooth catenin levels in cell differentiation decreased significantly until late differentiation of neural stem cells have recovered treat AD.

Wnt promotes proliferation and differentiation of neural stem cells, posterior catenin cells are activated, can promote transcription of cyclin D and other genes by binding to TCF within the nucleus, and thus play a role in promoting cell proliferation and maintenance of stem cell populations. Classic Wnt / sweat catenin signaling is mainly to promote the differentiation of neural stem cells, in particular to promote neuronal differentiation. Studies have shown that, Wnt signal can promote neural stem cells differentiate into neurons and astrocytes, and oligodendrocytes suppressed differentiation. The role of Wnt signaling pathway in neonatal mouse brain cortex neural stem cells, found in the cerebral cortex nerve cells express the receptor and signaling molecule Wnt proteins, and only for a specific area of neural stem cells in newborn period have an effect amount of proliferation and differentiation. Experimental results show that the component Wnt signaling pathways promoting neuronal differentiation by the action of inhibiting USK-3R, activation of sweat catenin transcription of target genes, and reduced apoptosis ${ }^{[3]}$.

\section{Treatment of Alzheimer's Disease by Wnt Pathway}

Neural stem cell transplantation for the treatment of AD is a clinical wealthy future prospects of treatment, its purpose is to repair and replace damaged nerve cells, cell reconstruction loop and cellular function, mainly through two ways: (1) endogenous way, that induce endogenous neural stem cell proliferation and differentiation, so that the damaged central nervous system to repair 
itself; (2) extrinsic pathway that directly replace tissue defect or implant genetically engineered cells. Exogenous neural stem cells, treatment of neurological disorders methods include cell transplantation and gene therapy [4].

Neural stem cells labeled in brau can be transplanted into the bilateral hippocampus dentate gyrus, found inside the cutting fimbria hippocampus density of transplanted cells were significantly higher than the normal side, and cut the hippocampus FIMBRIA side Nissl stained neuronal cell bodies of large sample cells significantly more than the normal side, neural stem cells can survive and coastal horse dentate granule lower migration. The mesenchymal stem cells into rats with bilateral hippocampal FF off dementia model, the results showed that the ability of learning and memory in rats in the cholinergic system partially improved. By exploring transplanted adipose-derived mesenchymal stem cells (A-MSC) treatment of AD, clearly found it to be a useful, have the potential and innovative treatments. $\mathrm{AD}$ transplantation therapy for $\mathrm{AD}$, but the biggest challenge is the lack of autologous stem cells.

Neural stem cells already have been widely used in transplant experimental study of various degenerative diseases of the nervous system. After transfection with p-EUFP-hTH marrow stromal derived neural stem cell transplantation can significantly improve symptoms of PD monkey, PET dopamine transporter imaging showed transplantation surgery striatum contralateral lower than the average standard uptake rate and healthy monkeys, and above PD monkey operative side, suggesting that transfection of neural stem cell transplantation enables stable expression of the gene of effective and play a therapeutic role in the host. Neural stem cells were successfully cultured had been confirmed, the above researchers found that transplanted neural stem cells, after energy survival, differentiation, and migration, the purpose of our group study is to explore the Wnt pathway modified neural stem cells obtained functional nerve cells transplanted into AD cognitive memory of their party and the improved situation of SD rats.

\section{Treatment of Alzheimer's Disease by Neural Stem Cell}

In recent years, with the success of neural stem cells in embryonic and adult mammalian central nervous system discovery and in vitro, longitudinal studies have shown that many of its unique biological characteristics, namely: undifferentiated, self-copy update, continue proliferation pluripotent mo, can migrate, low immunogenicity, and so integrated into the host brain. Neural stem cell transplantation for treatment of possible mechanisms of $\mathrm{AD}$ as follows: to differentiate into neural-like cells secrete neurotrophic factors, and promote endogenous repair, stimulate angiogenesis, the role of inflammatory cells less relevant. This is the treatment of AD provides a new way of thinking, is about in vitro amplification of neural stem cells transplanted into the brain to replace lost, damaged nerve cells and repair of the nervous system.

Neural stem cells are capable of self-replication, and can differentiate to produce neurons in certain microenvironment. Currently neural stem cells have made great progress in animal experiments. Applications immunotoxin 192-IgG-saporin SD injected into rat brain cholinergic system injury established Alzheimer's disease model, its basal ago neural stem cell transplantation. After the transplant, the medial septal nucleus injury side, diagonal band p75NGFR positive neurons in the count area, perimeter, gray were increased; SD rats learning and memory capacity than the previous improvements - transplantation of neural stem cells on 192-IgG The number of positive neurons p75NGFR before Alzheimer's disease model rat basal -saporin brain degeneration with complementary and protection.

Schwann cells can promote the proliferation of neural stem cells, and therefore in the treatment of Alzheimer's disease rat model, the Schwann cells and neural stem cells transplanted into Alzheimer's disease model with rat brain were observed for Alzheimer joint plant silent influence disease rats. It is clear that combined transplantation group has a significant effect on improving learning and memory abilities of rats with Alzheimer disease, and better than the transplantation of neural stem cells alone. Olfactory ensheathing cells can produce a variety of nerve stem cell proliferation and differentiation into neurons of neurotrophic factor, the olfactory ensheathing cells and neural stem cells implanted in rat brain together after transplantation, transplantation of neural 
stem cell survival group a larger number of nerve and stem cells into cholinergic neuron differentiation increased significantly.

Neural stem cells therapy certain degree of progress has been made in animal models of Alzheimer's disease, then took advantage of the characteristics of certain cells can nutrition neural stem cells, which neural stem cells transplantation for the treatment of Alzheimer's disease; also gene on neural stem cells have been modified to improve the function of neural stem cells, and enhance the efficacy of neural stem cells in the treatment of Alzheimer's disease. But now neural stem cell proliferation, differentiation, internal migration and the organizational structure of fused cells, external environmental regulation and control mechanism is unclear, after transplantation of neural stem cell migration speed, direction of differentiation lack of effective means of regulation, and the immunogen neural stem cells and strong, which makes it more difficult clinical application, and therefore the need to use weak immunogenicity stem cells to treat.

\section{Conclusions}

At present, although Alzheimer's disease has been progress in stem cell Cure, but the treatment of Alzheimer's disease is still the main drug treatment, we believe that stem cells can cure Alzheimer's such disease neurodegenerative diseases. Neural stem cells are a class derived from the nervous system, has the self-renewal capacity, can produce a variety of asymmetric division of neural cells pluripotent stem cells. Regulation of neural stem cells to differentiate into specific neurons become a difficult point, signal transduction plays an important role in the differentiation of neural stem cells and Wnt signaling pathway is an important extracellular factor of regulating the proliferation and differentiation of neural stem cells. Inactivation of Wnt signaling pathway promotes the progression of Alzheimer's disease, and activation of Wnt signaling pathway protects hippocampal neurons and promotes the differentiation of neural stem cells. This may provide novel therapeutic methods for Alzheimer's disease.

\section{Acknowledgment}

Subject Source: Qiqihaer Science and Techology Bureau.

\section{References}

[1] T Jessberger S, Gage FH. Fate plasticity of adult hippocampal progenitors: biological relevance and therapeutic use. Trends Pharmacol Sci. 2009; 30(2):61-65.

[2] Inestrosa NC. Arenas E. Emerging roles of Wnts in the adult nervous system. Nat Rev Neurosci. 2010; 11(2):77-86.

[3] Zilka N, Zilkova M, Kazmerova Z, et al. Mesenchymal stem cells rescue the Alzheimer's disease cell model from cell death induced by misfolded truncated tau. Neuroscience 2011:193: 330-337

[4] Wei LC, Ding YX, Liu YH, et al. Low-dose radiation stimulates Wnt/beta-catenin signaling, neural stem cell proliferation and neurogenesis of the mouse hippocampus in vitro and in vivo. CurrAlzheimer Res. 2012; 9(3):278-289.

[5] Chen BY, Wang X, Chen $\mathrm{L}$ w, et al. Molecular targeting regulation of proliferation and differentiation of the bone marrow-derived mesenchymal stem cells or mesenchymal stromal cells. Curr Drug Targets. 2012; 13(4):561-571. 